\title{
THE ASYMPTOTIC ANALYSIS OF THE INCOME IN CLOSED HM-STRUCTURE WITH PRIORITY MESSAGES
}

\author{
Mikhail Matalytski ${ }^{1}$, Olga Kiturko ${ }^{2}$ \\ ${ }^{I}$ Institute of Mathematics, Czestochowa University of Technology \\ Czestochowa, Poland \\ ${ }^{2}$ Faculty of Mathematics and Computer Science, Grodno State University \\ Grodno, Belarus \\ ${ }^{1}$ m.matalytski@gmail.com, ${ }^{2}$ sytaya_om@mail.ru
}

\begin{abstract}
In the article the asymptotic analysis of closed exponential queueing HM-structure with priority messages is carried out with a large total number of messages, depending on time. The number of service lines in systems, the intensity of service messages in them, the probabilities of message transitions between systems also depends on time. It is proved that the density of the income distribution in the network systems in asymptotic satisfies differential equations in partial derivatives. This provided the inhomogeneous differential equation for the expected incomes system structure. An example of transport logistics shows how to solve such equations.
\end{abstract}

\section{Introduction}

The concept of the closed queueing structure (CQS) with a central system of service has been introduced in monography [1]. In article [2] an asymptotic analysis CQS of arbitrary architecture with one-type messages and parametres, depending on the time, is carried out. In this article, such an analysis is carried out for CQS with priority messages.

Let's consider the closed exponential queueing structure with priority messages consisting of $n+1$ queueing systems $S_{0}, S_{1}, \ldots, S_{n}$, where $S_{0}$ - environment, as which condition we will understand a vector $k(t)=(k, t)=\left(k_{11}(t), k_{12}(t) ; k_{21}(t)\right.$, $\left.k_{22}(t) ; \ldots ; k_{n 1}(t), k_{n 2}(t)\right)$, where $k_{i 1}(t), k_{i 2}(t)$ - accordingly the number of priority and priority-free messages in system $S_{i}$ in an instant $t$. Priority messages have an absolute priority in relation to priority-free messages [3]. Let $K_{1}(t)$ and $K_{2}(t)$ respectively be the number of the priority and priority-free messages served in CQS, $K_{1}(t)+K_{2}(t)=K(t)$ - total number of served messages in an instant $t$. It is apparent that in system $S_{0}$ the number of priority and usual messages in an instant $t$ equal respectively $k_{01}(t)=K_{1}(t)-\sum_{i=1}^{n} k_{i 1}(t)$ and $k_{02}(t)=K_{2}(t)-\sum_{i=1}^{n} k_{i 2}(t)$. The number of service lines in queuing system (QS) $m_{i}(t), i=\overline{1, n}, m_{0}(t)=K(t)$, prob- 
abilities of messages transition between them $p_{i j}(t), i, j=\overline{0, n}$, depend on time. Disciplines of service of messages of both types in each QS are FIFO. Let's designate through $\mu_{j s}(t)$ - service intensity of messages of type $s$ in $j$-th QS in an instant $t$. Let's also enter the following designations:

$\varepsilon_{j 1}\left(k_{j 1}(t), m_{j}(t)\right)=\min \left\{k_{j 1}(t), m_{i}(t)\right\}$,
$\varepsilon_{j 2}\left(k_{j 1}(t), k_{j 2}(t), m_{j}(t)\right)=\left\{\begin{array}{l}k_{j 2}(t), k_{j 1}(t)+k_{j 2}(t)<m_{j}(t), \\ m_{j}(t)-k_{j 1}(t), k_{j 1}(t)<m_{j}(t), k_{j 1}(t)+k_{j 2}(t) \geq m_{j}(t), \\ 0, k_{j 1}(t) \geq m_{j}(t), j=\overline{0, n} .\end{array}\right.$

\section{Equation conclusion for density of distribution of the expected income of separate system}

Let's designate through $v_{c}^{*}(k, t)$ the full expected income which will receive QS $S_{c}$ of the closed structure with priority messages in time $t$, if in an initial timepoint of CQS is in a condition $k$. During a small period $\Delta t$ of CQS can remain in condition $(k, t)$ or make transition to condition $\left(k+I_{i 1}-I_{j 1}, t+\Delta t\right),\left(k+I_{i 2}-I_{j 2}, t+\Delta t\right)$, thus for simplicity we will consider that $p_{i i}(t)=0, i=\overline{0, n}$. Here $I_{0 s}$ - vector consisting of zero, $I_{i s}-2 n$-vector with zero components, except for a component with the number $2(i-1)+s$ which is equal to $1, i=\overline{1, n}, s=1,2$.

Theorem. Income distribution density $p_{v c}^{*}(x, t)$ under a condition that it is differentiated on $t$ and is twice sectionally continuous differentiated on $x_{i s}, x_{j s}$, $i, j=\overline{1, n}, s=1,2$, satisfies the terms of order of smallness $\varepsilon^{2}(t)$ to the following differential equation in partial derivatives:

$$
\begin{gathered}
\frac{\partial p_{v c}^{*}(x, t)}{\partial t}=-\sum_{i=1}^{n} \sum_{s=1}^{2} A_{i s}(x, t) \frac{\partial p_{v c}^{*}(x, t)}{\partial x_{i s}}+\frac{\varepsilon(t)}{2} \sum_{i, j=1 s=1}^{n} \sum_{i j s}^{2} B_{i s}(x, t) \frac{\partial^{2} p_{v c}^{*}(x, t)}{\partial x_{i s} \partial x_{j s}}- \\
-2 n K(t) \varepsilon^{\prime}(t) p_{v c}^{*}(x, t)+r_{c}^{*}(x, t),
\end{gathered}
$$

where

$$
\begin{gathered}
A_{i 1}(x, t)=\sum_{j=1}^{n} \mu_{j 1}(t) p_{j i}^{*}(t) \varepsilon_{j 1}\left(x_{j 1}, l_{j}(t)\right)+\mu_{01}(t) p_{0 i}(t)\left(\frac{K_{1}(t)}{K(t)}-\sum_{j=1}^{n} x_{j 1}\right), \\
A_{i 2}(x, t)=\sum_{j=1}^{n} \mu_{j 2}(t) p_{j i}^{*}(t) \varepsilon_{j 2}\left(x_{j 1}, x_{j 2}, l_{j}(t)\right)+\mu_{02}(t) p_{0 i}(t)\left(\frac{K_{2}(t)}{K(t)}-\sum_{j=1}^{n} x_{j 2}\right),
\end{gathered}
$$




$$
\begin{gathered}
B_{i j 1}(x, t)=-\mu_{j 1}(t) p_{j i}(t) \varepsilon_{j 1}\left(x_{j 1}, l_{j}(t)\right), B_{i i 1}(x, t)=\sum_{j=0}^{n} \mu_{j 1}(t) q_{j i}^{*}(t) \varepsilon_{j 1}\left(x_{j 1}, l_{j}(t)\right), \\
B_{i j 2}(x, t)=-\mu_{j 2}(t) p_{j i}(t) \varepsilon_{j 2}\left(x_{j 1}, x_{j 2}, l_{j}(t)\right), \\
B_{i i 2}(x, t)=\sum_{j=0}^{n} \mu_{j 2}(t) q_{j i}^{*}(t) \varepsilon_{j 2}\left(x_{j 1}, x_{j 2}, l_{j}(t)\right), \\
p_{j i}^{*}(t)=\left\{\begin{array}{l}
p_{j i}(t)-1, j=i, \\
p_{j i}(t), j \neq i,
\end{array} \quad q_{j i}^{*}(t)=\left\{\begin{array}{l}
1+p_{j i}(t), j=i, \\
p_{j i}(t), j \neq i,
\end{array}\right.\right. \\
r_{c}^{*}(x, t)=K(t) \sum_{i, j=0}^{n}\left[\mu_{j 1}(t) \varepsilon_{j 1}\left(x_{j 1}, l_{j}(t)\right) r_{j i c}^{(1)}(t)+\right. \\
\left.+\mu_{j 2}(t) \varepsilon_{j 2}\left(x_{j 1}, x_{j 2}, l_{j}(t)\right) r_{j i c}^{(2)}(t)\right] p_{j i}(t)+r_{c}(t), \\
r_{j i c}^{(s)}(t)=K^{n}(t) R_{j i c}^{(s)}(t), r_{c}(t)=K^{n}(t) R_{c}(t), i, j, c=1, n, s=1,2,
\end{gathered}
$$

income from transitions between conditions of CQS $R_{j i c}^{(s)}(t), R_{c}(t)$ is defined in the proof.

Proof.

Let's say that if on an interval of time $[t, t+\Delta t]$ of CQS makes transition from condition $(k, t)$ to condition $\left(k+I_{i 1}-I_{j 1}, t+\Delta t\right)$ (it can happen to probability $\left.\mu_{j 1}(t) \varepsilon_{j 1}\left(k_{j 1}(t), m_{j}(t)\right) u\left(k_{j 1}(t)\right) u\left(K_{1}(t)-k_{i 1}(t)\right) p_{j i}(t) \Delta t+o(\Delta t)\right)$, where $u(x)$ - function of Heaviside, the income of system $S_{c}$ will make $R_{j i 1}^{(1)}(t)$, therefore the income of this QS in an instant $t+\Delta t$ will be equal to this size plus the expected income $v_{c}^{*}\left(k+I_{i 1}-I_{j 1}, t\right)$ which it receives for the remaining time $t$ if the condition was initial $\left(k+I_{i 1}-I_{j 1}, t\right)$. Similarly, if on interval $[t, t+\Delta t]$ of CQS makes transition from condition $(k, t)$ to condition $\left(k+I_{i 2}-I_{j 2}, t+\Delta t\right)$ with probability $\mu_{j 2}(t) \varepsilon_{j 2}\left(k_{j 1}(t), k_{j 2}(t), m_{j}(t)\right) u\left(k_{j 2}(t)\right) u\left(K_{2}(t)-k_{i 2}(t)\right) p_{j i}(t) \Delta t+o(\Delta t)$, the income of system $S_{c}$ will make $R_{j i 1}^{(2)}(t)$ plus the expected income $v_{c}^{*}\left(k+I_{i 2}-I_{j 2}, t\right)$ which it receives for the remaining time $t$ if the condition was initial $\left(k+I_{i 2}-I_{j 2}, t\right)$. Let's consider besides, that the system $S_{c}$ receives income $R_{c}(t)$ for a unit of time during the stay of CQS in condition $(k, t)$. CQS remains in condition $(k, t)$ during time $\Delta t$ with probability $1-\sum_{j=0}^{n}\left[\mu_{j 1}(t) \varepsilon_{j 1}\left(k_{j 1}(t), m_{j}(t)\right)+\mu_{j 2}(t) \varepsilon_{j 2}\left(k_{j 1}(t), k_{j 2}(t), m_{j}(t)\right)\right] \times$ $\times \Delta t+o(\Delta t)$, thus the income of system $S_{c}$ will make $R_{c}(t) \Delta t+v_{c}^{*}(k, t)$. 
Owing to the aforesaid, the full expected income $v_{c}^{*}(k, t+\Delta t)$ of system $S_{c}$ in an instant $t+\Delta t$ satisfies the system of the difference equations:

$$
\begin{gathered}
v_{c}^{*}(k, t+\Delta t)=\left\{1-\sum_{j=0}^{n}\left[\mu_{j 1}(t) \varepsilon_{j 1}\left(k_{j 1}(t), m_{j}(t)\right)+\mu_{j 2}(t) \varepsilon_{j 2}\left(k_{j 1}(t), k_{j 2}(t), m_{j}(t)\right)\right] \Delta t\right\} \times \\
\times\left(R_{c}(t) \Delta t+v_{c}^{*}(k, t)\right)+\sum_{i, j=0}^{n}\left[\mu_{j 1}(t) \varepsilon_{j 1}\left(k_{j 1}(t), m_{j}(t)\right) u\left(k_{j 1}(t)\right) u\left(K_{1}(t)-k_{i 1}(t)\right) p_{j i}(t) \Delta t \times\right. \\
\times\left(R_{j i c}^{(1)}(t)+v_{c}^{*}\left(k+I_{i 1}-I_{j 1}, t\right)\right)+\mu_{j 2}(t) \varepsilon_{j 2}\left(k_{j 1}(t), k_{j 2}(t), m_{j}(t)\right) u\left(k_{j 2}(t)\right) \times \\
\left.\times u\left(K_{2}(t)-k_{i 2}(t)\right) p_{j i}(t) \Delta t\left(R_{j i c}^{(2)}(t)+v_{c}^{*}\left(k+I_{i 2}-I_{j 2}, t\right)\right)\right]+o(\Delta t) .
\end{gathered}
$$

Owing to the expression definition $\varepsilon_{j 1}, \varepsilon_{j 2}$, according to (1) and to definition of function of Heaviside, in the right part of a relation (6) we can lower functions $u\left(k_{j 1}(t)\right), u\left(k_{j 2}(t)\right)$. Besides, hereinafter we will carry out the asymptotic analysis at $K_{s}(t) \leq N \rightarrow \infty, s=1,2$, therefore it is possible to consider that $u\left(K_{1}(t)-k_{i 1}(t)\right)=$ $=u\left(K_{2}(t)-k_{i 2}(t)\right)=1$. Considering $\Delta t \rightarrow 0$ from (6) we receive a system of difference-differential equations (DDE) for expected incomes of system $S_{c}$ :

$$
\begin{gathered}
\frac{d v_{c}^{*}(k, t)}{d t}=\sum_{i, j=0}^{n}\left\{\left[\mu_{j 1}(t) \varepsilon_{j 1}\left(k_{j 1}(t), m_{j}(t)\right)\left(v_{c}^{*}\left(k+I_{i 1}-I_{j 1}, t\right)-v_{c}^{*}(k, t)\right)+\right.\right. \\
\left.\left.+\mu_{j 2}(t) \varepsilon_{j 2}\left(k_{j 1}(t), k_{j 2}(t), m_{j}(t)\right)\left(v_{c}^{*}\left(k+I_{i 2}-I_{j 2}, t\right)-v_{c}^{*}(k, t)\right)\right] p_{j i}(t)\right\}+ \\
+\sum_{i, j=0}^{n}\left[\mu_{j 1}(t) \varepsilon_{j 1}\left(k_{j 1}(t), m_{j}(t)\right) R_{j i c}^{(1)}(t)+\right. \\
\left.+\mu_{j 2}(t) \varepsilon_{j 2}\left(k_{j 1}(t), k_{j 2}(t), m_{j}(t)\right) R_{j i c}^{(2)}(t)\right] p_{j i}(t)+R_{c}(t)
\end{gathered}
$$

Let's pass to density of distribution of the income of QS $S_{c} p_{v c}^{*}(x, t)$. Considering a case of a large number of messages $1<<(t)<N$ and passing to a vector of relative variables

$$
\xi^{*}(t)=\left(\frac{k_{11}(t)}{K(t)}, \frac{k_{12}(t)}{K(t)} ; \frac{k_{21}(t)}{K(t)}, \frac{k_{22}(t)}{K(t)} ; \ldots ; \frac{k_{n 1}(t)}{K(t)}, \frac{k_{n 2}(t)}{K(t)}\right),
$$

whose possible values belong to the limited closed set

$$
G^{*}=\left\{x=\left(x_{11}, x_{12} ; x_{21}, x_{22} ; \ldots ; x_{n 1}, x_{n 2}\right): x_{i s} \geq 0, s=1,2 ; \sum_{i=1}^{n}\left(x_{i 1}+x_{i 2}\right) \leq 1\right\},
$$

in which they place in knots of $2 n$-dimensional lattice on distance $\varepsilon(t)$ from each other, we can use function approximation $v_{c}^{*}(k, t): K^{2 n}(t) v_{c}^{*}(k, t)=$ 
$=K^{2 n}(t) v_{c}^{*}(x K(t), t)=p_{v c}^{*}(x, t)$, where $p_{v c}^{*}(x, t)$ - density of distribution of probabilities of a vector $\xi^{*}(t)$.

Let $e_{i 1}=\varepsilon(t) I_{i 1}, e_{i 2}=\varepsilon(t) I_{i 2}, i=\overline{1, n}$, and let at $K(t) \rightarrow \infty$ :

$$
K^{2 n}(t) R_{j i c}^{(1)}(t)=r_{j i c}^{(1)}(t), K^{2 n}(t) R_{j i c}^{(2)}(t)=r_{j i c}^{(2)}(t), K^{2 n}(t) R_{c}(t)=r_{c}(t) .
$$

Let's increase both parts (7) on $K^{2 n}(t)$ and having added to both parts $2 n K^{2 n-1}(t) K^{\prime}(t) v_{c}^{*}(k, t)=2 n K^{-1}(t) K^{\prime}(t) p_{v c}^{*}(x, t)=2 n \varepsilon(t) K^{\prime}(t) p_{v c}^{*}(x, t)=-2 n K(t) \times$ $\times \varepsilon^{\prime}(t) p_{v c}^{*}(x, t)$, we receive

$$
\begin{aligned}
& \frac{\partial p_{v c}^{*}(x, t)}{\partial t}=-2 n K(t) \varepsilon^{\prime}(t) p_{v c}^{*}(x, t)+K(t) \sum_{i, j=0}^{n}\left\{\left[\mu_{j 1}(t) \varepsilon_{j 1}\left(x_{j 1}(t), l_{j}(t)\right) \times\right.\right. \\
& \times\left(p_{v c}^{*}\left(x+e_{i 1}-e_{j 1}, t\right)-p_{v c}^{*}(x, t)\right)+\mu_{j 2}(t) \varepsilon_{j 2}\left(x_{j 1}(t), x_{j 2}(t), l_{j}(t)\right) \times \\
&\left.\left.\times\left(p_{v c}^{*}\left(x+e_{i 2}-e_{j 2}, t\right)-p_{v c}^{*}(x, t)\right)\right] p_{j i}(t)\right\}+r_{c}^{*}(x, t) .
\end{aligned}
$$

Let's spread out functions $p_{v c}^{*}\left(x+e_{i s}-e_{j s}, t\right), p_{v c}^{*}\left(x+e_{0 s}-e_{j s}, t\right)=p_{v c}^{*}\left(x-e_{j s}, t\right)$, $p_{v c}^{*}\left(x+e_{i s}-e_{0, s}, t\right)=p_{v c}^{*}\left(x+e_{i s}, t\right)$ abreast Taylor in a point neighbourhood $(x, t)$ :

$$
\begin{gathered}
p_{v c}^{*}\left(x+e_{i s}-e_{j s}, t\right)=p_{v c}^{*}(x, t)+\varepsilon(t)\left(\frac{\partial p_{v c}^{*}(x, t)}{\partial x_{i s}}-\frac{\partial p_{v c}^{*}(x, t)}{\partial x_{j s}}\right)+ \\
+\frac{\varepsilon^{2}(t)}{2}\left(\frac{\partial^{2} p_{v c}^{*}(x, t)}{\partial x_{i s}^{2}}-2 \frac{\partial^{2} p_{v c}^{*}(x, t)}{\partial x_{i s} \partial x_{j s}}+\frac{\partial^{2} p_{v c}^{*}(x, t)}{\partial x_{j s}^{2}}\right)+o\left(\varepsilon^{2}(t)\right), \\
p_{v c}^{*}\left(x-e_{j s}, t\right)=p_{v c}^{*}(x, t)-\varepsilon(t) \frac{\partial p_{v c}^{*}(x, t)}{\partial x_{j s}}+\frac{\varepsilon^{2}(t)}{2} \frac{\partial^{2} p_{v c}^{*}(x, t)}{\partial x_{j s}^{2}}+o\left(\varepsilon^{2}(t)\right), \\
p_{v c}^{*}\left(x+e_{i s}, t\right)=p_{v c}^{*}(x, t)+\varepsilon(t) \frac{\partial p_{v c}^{*}(x, t)}{\partial x_{i s}}+\frac{\varepsilon^{2}(t)}{2} \frac{\partial^{2} p_{v c}^{*}(x, t)}{\partial x_{i s}^{2}}+o\left(\varepsilon^{2}(t)\right), \\
i, j, c=\overline{0, n}, s=1,2 .
\end{gathered}
$$

Having substituted this decomposition in the equation (9):

$$
\begin{aligned}
\frac{\partial p_{v c}^{*}(x, t)}{\partial t} & =\sum_{i=1}^{n}\left[\sum _ { j = 1 } ^ { n } \left\{\mu _ { j 1 } ( t ) p _ { j i } ( t ) \varepsilon _ { j 1 } ( x _ { j 1 } ( t ) , l _ { j } ( t ) ) \left[\left(\frac{\partial p_{v c}^{*}(x, t)}{\partial x_{i 1}}-\frac{\partial p_{v c}^{*}(x, t)}{\partial x_{j 1}}\right)+\right.\right.\right. \\
& \left.\left.+\frac{\varepsilon(t)}{2}\left(\frac{\partial^{2} p_{v c}^{*}(x, t)}{\partial x_{i 1}^{2}}-2 \frac{\partial^{2} p_{v c}^{*}(x, t)}{\partial x_{i 1} \partial x_{j 1}}+\frac{\partial^{2} p_{v c}^{*}(x, t)}{\partial x_{j 1}^{2}}\right)\right]\right\}+
\end{aligned}
$$




$$
\begin{aligned}
& +\mu_{01}(t) p_{0 i}(t)\left(\frac{K_{1}(t)}{K(t)}-\sum_{j=1}^{n} x_{j 1}\right)\left(\frac{\partial p_{v c}^{*}(x, t)}{\partial x_{i 1}}+\frac{\varepsilon(t)}{2} \frac{\partial^{2} p_{v c}^{*}(x, t)}{\partial x_{i 1}^{2}}\right)+ \\
& +\sum_{j=1}^{n}\left\{\mu _ { j 2 } ( t ) p _ { j i } ( t ) \varepsilon _ { j 2 } ( x _ { j 1 } ( t ) , x _ { j 2 } ( t ) , l _ { j } ( t ) ) \left[\left(\frac{\partial p_{v c}^{*}(x, t)}{\partial x_{i 2}}-\frac{\partial p_{v c}^{*}(x, t)}{\partial x_{j 2}}\right)+\right.\right. \\
& \left.\left.+\frac{\varepsilon(t)}{2}\left(\frac{\partial^{2} p_{v c}^{*}(x, t)}{\partial x_{i 2}^{2}}-2 \frac{\partial^{2} p_{v c}^{*}(x, t)}{\partial x_{i 2} \partial x_{j 2}}+\frac{\partial^{2} p_{v c}^{*}(x, t)}{\partial x_{j 2}^{2}}\right)\right]\right\}+ \\
& \left.+\mu_{02}(t) p_{02}(t)\left(\frac{K_{2}(t)}{K(t)}-\sum_{j=1}^{n} x_{j 2}\right)\left(\frac{\partial p_{v c}^{*}(x, t)}{\partial x_{i 2}}+\frac{\varepsilon(t)}{2} \frac{\partial^{2} p_{v c}^{*}(x, t)}{\partial x_{j 2}^{2}}\right)\right]+ \\
& +\sum_{j=1}^{n}\left\{\mu_{j 1}(t) p_{j 0}(t) \varepsilon_{j 1}\left(x_{j 1}(t), l_{j}(t)\right)\left[-\frac{\partial p_{v c}^{*}(x, t)}{\partial x_{j 1}}+\frac{\varepsilon(t)}{2} \frac{\partial^{2} p_{v c}^{*}(x, t)}{\partial x_{j 1}^{2}}\right]\right\}+ \\
& +\sum_{j=1}^{n}\left\{\mu _ { j 2 } ( t ) p _ { j 0 } ( t ) \varepsilon _ { j 2 } ( x _ { j 1 } ( t ) , x _ { j 2 } ( t ) , l _ { j } ( t ) ) \left[-\frac{\partial p_{v c}^{*}(x, t)}{\partial x_{j 2}}+\right.\right. \\
& \left.\left.+\frac{\varepsilon(t)}{2} \frac{\partial^{2} p_{v c}^{*}(x, t)}{\partial x_{j 2}^{2}}\right]\right\}-2 n K(t) \varepsilon^{\prime}(t) p_{v c}^{*}(x, t)+r_{c}^{*}(x, t) \text {. }
\end{aligned}
$$

Using designations (3)-(5), the last equation to within members of order $O\left(\varepsilon^{2}(t)\right)$ can be written down in the form of (2) Towers has proven.

Let's notice, as appears from (4), (5) that $\frac{\varepsilon(t)}{2} \sum_{i, j=1}^{n} B_{i j s}(x, t) \frac{\partial^{2} p_{v c}^{*}(x, t)}{\partial x_{i s} \partial x_{j s}}=O\left(\varepsilon^{2}(t)\right)$, therefore from (2) follows that to within $\varepsilon^{2}(t)$ :

$$
\frac{\partial p_{v c}^{*}(x, t)}{\partial t}=-\sum_{i=1}^{n} \sum_{s=1}^{2} A_{i s}(x, t) \frac{\partial p_{v c}^{*}(x, t)}{\partial x_{i s}}-2 n K(t) \varepsilon^{\prime}(t) p_{v c}^{*}(x, t)+r_{c}^{*}(x, t) .
$$

\section{Finding of the expected income of separate systems}

Let's integrate both members of equation (10) on $x$ in area $G^{*}$ and we will divide both parts into the volume of the area $G^{*}$ equal $m\left(G^{*}\right)$ :

$$
\begin{gathered}
\frac{1}{m\left(G^{*}\right)} \iint_{G^{*}} \ldots \int \frac{\partial p_{v c}^{*}(x, t)}{\partial t} d x=-\frac{1}{m\left(G^{*}\right)} \sum_{i=1}^{n} \sum_{s=1}^{2} \iint_{G^{*}} \ldots \int A_{i s}(x, t) \frac{\partial p_{v c}^{*}(x, t)}{\partial x_{i s}} d x- \\
-\frac{2 n K(t) \varepsilon^{\prime}(t)}{m\left(G^{*}\right)} \iint_{G^{*}} \ldots \int p_{v c}^{*}(x, t) d x+\frac{1}{m\left(G^{*}\right)} \iint_{G^{*}} \ldots \int r_{c}^{*}(x, t) d x .
\end{gathered}
$$


Let's designate through $\bar{v}_{G^{*} c}^{p r}(t)$ - an average on $x$ value of the income of system $S_{c}$ CQS with priority messages on condition of change of an initial condition $x$ in area $G^{*}$. Having applied to both parts (11) of a reasoning as in article [2], we will receive the following equation for the average income of system $S_{c}$ :

$$
\frac{d}{d t} \bar{v}_{G^{*} c}^{p r}(t)=\left[\sum_{i=1}^{n} \sum_{s=1}^{2} \frac{\partial A_{i s}(x, t)}{\partial x_{i s}}-2 n K(t) \varepsilon^{\prime}(t)\right] \bar{v}_{G^{*} c}^{p r}(t)+\frac{1}{m\left(G^{*}\right)} \iint_{G^{*}} \ldots \int r_{c}^{*}(x, t) d x .
$$

From (3) follows that the coefficient $A_{i 1}(x, t)$ is piecewise and linear on $x_{j 1}$, $j=\overline{1, n}$, function, and coefficient $A_{i 2}(x, t)$ - piecewise and linear on $x_{j 1}, x_{j 2}, j=\overline{1, n}$, function, therefore (12) is DE with an explosive right part. It is possible to solve it by splitting of phase space $G^{*}$ into a number of areas depending on what the values accept $x_{j 1}, x_{j 2}, j=\overline{1, n}$, and solutions of the equation in each area.

Let's note that the total expected income of CQS can be found as the sum of the income of all QS.

\section{Example}

The Transport Enterprise (TE) (system $S_{2}$ ) having a large number of cars (messages), sends them for transportation and acceptance of freights to various cities (environment, system $S_{0}$ ) and then they come back to the TE base, are unloaded in a warehouse (system $S_{1}$ ), receive a new task and the process proceeds similarly. That stayed freights can be prime value or perishable, or, for example, arriving from abroad on railway transport (thus structures have to be rather returned to the sender), certain messages can have a priority at service (unloading - loading, paperwork) therefore when forecasting the income in such a situation the results of this section can be applied.

The condition of CQS in this case is described by a vector $k(t)=(k, t)=\left(k_{11}(t), k_{12}(t) ; k_{21}(t), k_{22}(t)\right), \quad k_{01}(t)=K_{1}(t)-k_{11}(t)-k_{21}(t), \quad k_{02}(t)=$ $=K_{2}(t)-k_{12}(t)-k_{22}(t)$ - the number of priority and low-priority messages in system $S_{0}$. The matrix of probabilities of transitions looks like

$$
P(t)=\left\|p_{i j}(t)\right\|_{3 \times 3}=\left(\begin{array}{ccc}
0 & 1 & 0 \\
0 & 0 & 1 \\
1 & 0 & 0
\end{array}\right) .
$$

For a unit of time we will take one week. Practical experience showed that it is convenient to set the CQS parameters in the form of periodic functions of type 


$$
\begin{aligned}
& K_{s}(t)=a_{s}+\frac{b_{s}}{c \sin \left(\frac{2 \pi t}{10}\right)+d}, s=1,2, \quad K(t)=a_{1}+a_{2}+\frac{b_{1}+b_{2}}{c \sin \left(\frac{2 \pi t}{10}\right)+d}, \\
& \mu_{j s}(t)=a_{j s}+\frac{b_{j s}}{c_{j s} \sin \left(\frac{2 \pi t}{10}\right)+d_{j s}}, j=\overline{0,2}, s=1,2, \\
& m_{j}(t)=m_{j 1}+\frac{m_{j 2}}{m_{j 3} \sin \left(\frac{2 \pi t}{10}\right)+m_{j 4}}, j=1,2 \text {, } \\
& R_{j i c}^{(s)}(t)=R_{j i c 1}^{(s)}+\frac{R_{j i c 2}^{(s)}}{R_{j i c 3}^{(s)} \sin \left(\frac{2 \pi t}{10}\right)+R_{j i c 4}^{(s)}}, j=\overline{0,2}, s=1,2, \\
& R_{j}(t)=R_{j 1}+\frac{R_{j 2}}{R_{j 3} \sin \left(\frac{2 \pi t}{10}\right)+R_{j 4}}, j=1,2 \text {. }
\end{aligned}
$$

Let's find the solution of the equation (12) in area

$$
G_{1}^{*}=\left\{x: l_{1}(t)<x_{1 s^{\prime}} \leq 1, l_{2}(t)<x_{2 s^{\prime}} \leq 1, \sum_{s=1}^{2}\left(x_{1 s}+x_{2 s}\right) \leq 1\right\},
$$

Let's set coefficients in expressions (13) in a look: $a_{1}=50, a_{2}=60, b_{1}=11, b_{2}=9$, $c=10, d=20, a_{01}=0.0015, a_{02}=0.001, a_{11}=0.0025, a_{12}=0.0013, a_{21}=0.0015$, $a_{22}=0.0020, \quad b_{01}=2, \quad b_{02}=b_{11}=b_{12}=b_{21}=b_{22}=1, \quad c_{01}=3, \quad c_{02}=c_{11}=c_{12}=4$, $c_{21}=c_{22}=5, \quad d_{01}=d_{12}=d_{22}=7, \quad d_{02}=d_{21}=8, \quad d_{11}=6, \quad m_{11}=m_{22}=m_{23}=3$, $m_{12}=m_{13}=4, m_{14}=7, m_{21}=2, m_{24}=8, R_{11}=-2, R_{21}=-3, R_{12}=R_{13}=4, R_{22}=2$, $R_{23}=3, \quad R_{14}=R_{24}=10, \quad R_{1221}^{(1)}=-2, \quad R_{1222}^{(1)}=R_{2022}^{(2)}=1, \quad R_{1223}^{(1)}=R_{1224}^{(2)}=R_{2024}^{(2)}=5$, $R_{1224}^{(1)}=7, R_{2021}^{(1)}=R_{2023}^{(1)}=R_{1222}^{(2)}=R_{2023}^{(2)}=3, R_{2024}^{(1)}=10, R_{1221}^{(2)}=-3, R_{2022}^{(1)}=R_{1223}^{(2)}=4$. Then, solving equation (12) at starting conditions $\bar{v}_{G^{*}{ }_{2}}^{p r}(0)=1000$, it is possible to determine the expected income of system $S_{2}$ at a change of reference state by area $G^{*}$. This differential equation decided in a Mathematica package a Runge-Kutta method of the fourth order. Dependence of the expected income of system $S_{2}$ on time is presented in Figure 1. 


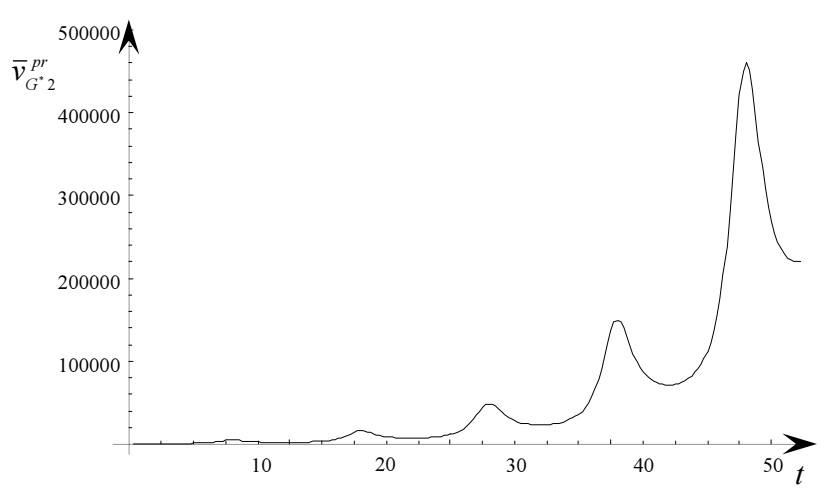

Fig. 1. Expected income of system $S_{2}$

\section{References}

[1] Matalytski M., Rusilko T., Mathematical analysis of stochastic models for claim processing in insurance companies, GrSU, Grodno 2007 (in Russian).

[2] Kiturko O., Matalytski M., Rusilko T., Asymptotic analysis of the total expected income of closed queueing structure with the one-type messages and application, Wiestnik GrUP 2013, 1(2) (in Russian).

[3] Tikhonov V., Mironov V., Markov Processes, Sov. Radio, Moscow 1977 (in Russian). 